\title{
Photometric Detection of Multiple Populations in Globular Clusters Using Integrated Light
}

\author{
William P. Bowman ${ }^{1,2,3}$ (1) Catherine A. Pilachowski ${ }^{1}$ (i), Liese van Zee ${ }^{1}$, Amanda Winans ${ }^{1}$, \\ Robin Ciardullo ${ }^{2,3}$ (1) , and Caryl Gronwall ${ }^{2,3}$ \\ ${ }^{1}$ Department of Astronomy, Indiana University, Bloomington, IN 47405, USA; bowman@psu.edu \\ 2 Department of Astronomy \& Astrophysics, The Pennsylvania State University, University Park, PA 16802, USA \\ Received 2017 May 10; revised 2017 July 22; accepted 2017 August 3; published 2017 September 5
}

\begin{abstract}
We investigate the multiple stellar populations of the globular clusters (GCs) M3, M5, M13, and M71 using $g^{\prime}$ and intermediate-band $\mathrm{CN}-\lambda 3883$ photometry obtained with the WIYN $0.9 \mathrm{~m}$ telescope on Kitt Peak. We find a strong correlation between red giant stars' $\mathrm{CN}-g^{\prime}$ colors and their spectroscopic sodium abundances, thus demonstrating the efficacy of the two-filter system for stellar population studies. In all four clusters, the observed spread in red giant branch $\mathrm{CN}-g^{\prime}$ colors is wider than that expected from photometric uncertainty, confirming the well-known chemical inhomogeneity of these systems. M3 and M13 show clear evidence for a radial dependence in the CNband strengths of its red giants, while the evidence for such a radial dependence of CN strengths in M5 is ambiguous. Our data suggest that the dynamically old, relatively metal-rich M71 system is well mixed, as it shows no evidence for chemical segregation. Finally, we measure the radial gradients in the integrated $\mathrm{CN}-\mathrm{g}^{\prime}$ color of the clusters and find that such gradients are easily detectable in the integrated light. We suggest that photometric observations of color gradients within GCs throughout the Local Group can be used to characterize their multiple populations, and thereby constrain the formation history of GCs in different galactic environments.
\end{abstract}

Key words: globular clusters: general - globular clusters: individual (M3, M5, M13, M71) - stars: abundances

Supporting material: data behind figure

\section{Introduction}

For most of the twentieth century, Milky Way globular clusters (GCs) were thought of as simple stellar populations, defined by a single age and metallicity. However, in the past several years, our view of these systems has changed, and it is now widely accepted that GCs contain multiple stellar populations (e.g., Bedin et al. 2004; Villanova et al. 2007; Carretta et al. 2010; Gratton et al. 2012; Milone et al. 2012; Piotto et al. 2015). Spectroscopic analyses have found that significant variations in the abundances of light elements, especially those associated with proton-capture processes, such as C, N, O, F, Na, Mg, and $\mathrm{Al}$ (see Gratton et al. 2012, and references therein) exist within most clusters. The physical mechanism responsible for these differences is still uncertain, with possible sources being the CNO-processed winds of massive, fast-rotating stars formed early in the history of the cluster and the superwinds of intermediate-mass asymptotic giant branch (AGB) stars (Gratton et al. 2012). Regardless of the cause, the dispersions in the abundances of these light elements, which have been measured in a number of GCs, are thought to indicate that GCs are more than simply single-age, single-metallicity stellar systems.

The most secure way of detecting abundance variations in the stars of GCs is through careful spectroscopic analyses. However, the presence of multiple stellar populations within a GC can also be revealed photometrically by examining features in the color-magnitude diagram (CMD), such as bifurcations in the main sequence (e.g., Milone et al. 2015) and red giant branch (RGB; e.g., Kayser et al. 2008; Milone et al. 2016). Numerous investigations of this type have been performed in

\footnotetext{
3 Institute for Gravitation and the Cosmos, The Pennsylvania State University, University Park, PA 16802, USA.
}

the past few years (e.g., Roh et al. 2011; Lee et al. 2013; Hsyu et al. 2014; Milone et al. 2017), though, with a few notable exceptions (e.g., Cummings et al. 2014; Lee 2015), the filter sets used were not optimized for abundance studies. This is unfortunate, because, as Sbordone et al. (2011) have demonstrated, filter systems such as those defined by Strömgren (1966) can greatly increase the sensitivity of photometry to chemical inhomogeneities. Such observations open up the possibility of studying abundance dispersions within the GCs of other galaxies, thus allowing studies of the physics of GC formation under conditions far different from those that existed in the Milky Way.

Spectroscopic studies of nearby GCs have found that sodium abundance correlates with stellar population, with second generation (SG) stars having higher abundances (Carretta et al. 2010). Because sodium often correlates with carbon and nitrogen (Gratton et al. 2012), one should be able to use CNband strengths as a tracer of stellar population. For example, Smith \& Langland-Shula (2009), Smith et al. (2013), and Smith (2015a, 2015b) have used literature measurements of spectroscopically determined $\mathrm{CN}, \mathrm{O}$, and $\mathrm{Na}$ line strengths to demonstrate a clear correlation between $\mathrm{CN}$ and $[\mathrm{Na} / \mathrm{Fe}]$ in the GCs NGC 288, NGC 362, M5, 47 Tucanae, and M71. These studies also found significant variations in the CN-band strength within the clusters, consistent with the results of Smith \& Briley (2006) and Martell \& Smith (2009).

Here, we examine the distribution of stellar populations within intermediate-metallicity GCs using narrow-band photometry centered on the $\mathrm{CN}$ absorption complex at $\lambda \sim 3883 \AA$. In Sections 2 and 3, we describe our photometric observations of four well-known Galactic GCs and detail the reduction techniques used to measure $\mathrm{CN}-g^{\prime}$ colors for the systems' RGB stars. In Section 4, we discuss the correlations between 
Table 1

Cluster Parameters

\begin{tabular}{|c|c|c|c|c|c|c|c|c|}
\hline Cluster & $M_{V}^{\mathrm{a}}$ & $\begin{array}{l}\text { Distance }^{\mathrm{a}} \\
\quad(\mathrm{kpc})\end{array}$ & $\begin{array}{c}\text { Mass }^{\mathrm{b}} \\
\left(10^{5} M_{\odot}\right)\end{array}$ & {$[\mathrm{Fe} / \mathrm{H}]^{\mathrm{a}}$} & $E(B-V)^{\mathrm{a}}$ & $\begin{array}{l}t\left(r_{\mathrm{h}}\right)^{\mathrm{c}} \\
(\mathrm{Gyr})\end{array}$ & $\begin{array}{l}\text { Half-light Radius, } r_{\mathrm{e}}{ }^{\mathrm{a}} \\
\text { (arcmin) }\end{array}$ & $\begin{array}{l}\text { Turnoff }^{\mathrm{d}} \\
u^{\prime}-g^{\prime}\end{array}$ \\
\hline M3 & -8.9 & 10.2 & 5.7 & -1.50 & 0.01 & 6.2 & 2.31 & 1.0 \\
\hline M5 & -8.8 & 7.5 & 5.7 & -1.29 & 0.03 & 2.6 & 1.77 & 1.0 \\
\hline M13 & -8.5 & 7.1 & 5.6 & -1.53 & 0.02 & 2.0 & 1.69 & 0.7 \\
\hline M71 & -5.6 & 4.0 & 4.4 & -0.78 & 0.25 & 0.27 & 1.67 & 1.5 \\
\hline
\end{tabular}

Notes.

${ }^{a}$ From Harris (2010).

${ }^{\mathrm{b}}$ From VandenBerg et al. (2013).

${ }^{\mathrm{c}}$ From Kimmig et al. (2015) and Kamann et al. (2014).

${ }^{\mathrm{d}}$ From An et al. (2008).

$\mathrm{CN}-g^{\prime}$ colors and $[\mathrm{Na} / \mathrm{Fe}]$ abundance, and in Section 5 , we characterize the photometric signatures of these abundance variations. In Section 6, we examine the behavior of the $\mathrm{CN}-g^{\prime}$ colors of GC integrated light and show that stochastic effects associated with stellar evolution are generally unimportant for such a measurement. We conclude by summarizing our results and their applicability to studies of multiple populations in GCs in nearby galaxies.

\section{Target Selection}

We chose as the targets for our study four nearby GCs that have a host of spectroscopic abundance measurements. Three of the clusters, M3, M5, and M13, are dynamically young $\left(t\left(r_{h}\right) \geqslant 2\right.$ Gyr $)$, bright $\left(M_{V}<-8.8\right)$, intermediate-metallicity $([\mathrm{Fe} / \mathrm{H}] \lesssim-1.3)$ systems with little foreground reddening $(E(B-V) \lesssim 0.03)$; the fourth (M71), is a relatively faint $\left(M_{V} \sim-5.6\right)$, nearby cluster $(d \sim 4 \mathrm{kpc})$, that has a shorter median relaxation time $\left(t\left(r_{h}\right)=0.27 \mathrm{Gyr}\right)$, higher metallicity $([\mathrm{Fe} / \mathrm{H}] \sim-0.8)$, and greater foreground extinction $(E(B-V)=0.25)$; see Table 1. All four systems have mass-to-light ratios $\left(M / L_{V} \leqslant 2.5\right)$ that confirm their identity as true GCs, rather than accreted dwarf galaxies (Kamann et al. 2014; Kimmig et al. 2015). Table 1 summarizes some of the relevant cluster parameters, along with the $u^{\prime}-g^{\prime}$ color of the main-sequence turnoff, as derived from Sloan Digital Sky Survey (SDSS) photometry (An et al. 2008). We use the latter values to establish the zero point of the instrumental magnitudes.

\section{Observations and Data Processing}

Images of the four program GCs were obtained using the Half Degree Imager (HDI) on the $f / 7.5$ WIYN 0.9 m telescope at Kitt Peak with both a Sloan $g^{\prime}$ filter and a $70 \AA$-wide interference filter centered on the $\mathrm{CN}$ absorption trough at $3883 \AA$. The image scale of the data is 0 ". 43 per pixel and the images typically have a full width at half maximum of $\sim 3.5$ pixels. The observations are summarized in Table 2 .

The data were processed using standard IRAF ${ }^{4}$ photometric $\mathrm{CCD}$ reduction techniques. After bias subtraction, flatfielding, and cosmic-ray rejection, the frames of each cluster were median-combined to create a high signal-to-noise pair of $\mathrm{CN}$ and $g^{\prime}$ images. An astrometric solution to each field was then obtained using the coordinates of reference stars in the

\footnotetext{
4 IRAF is distributed by the National Optical Astronomy Observatory, which is operated by the Association of Universities for Research in Astronomy (AURA) under a cooperative agreement with the National Science Foundation.
}

Table 2

Table of Observations

\begin{tabular}{|c|c|c|c|c|c|}
\hline Cluster & Filter & $\begin{array}{l}\text { Number of } \\
\text { Exposures }\end{array}$ & $\begin{array}{l}\text { Exp. Time } \\
\text { (s) }\end{array}$ & Seeing & Date \\
\hline M3 & $\mathrm{CN}$ & 3 & 900 & 1." 5 & $2015 \mathrm{Jul} 7$ \\
\hline M3 & $g^{\prime}$ & 5 & 30 & $1 . ! 2$ & $2015 \mathrm{Jul} 4$ \\
\hline M5 & $\mathrm{CN}$ & 3 & 900 & 1." 4 & 2015 Jul 7 \\
\hline M5 & $g^{\prime}$ & 10 & 30 & $2 . ! 1$ & 2015 Jul 6 \\
\hline M13 & $\mathrm{CN}$ & 4 & 900 & $1 . ! 7$ & 2015 Jul 6 \\
\hline M13 & $g^{\prime}$ & 5 & 30 & $1 . " 7$ & $2015 \mathrm{Jul} 4$ \\
\hline M71 & $\mathrm{CN}$ & 3 & 900 & $1 . " 6$ & $2015 \mathrm{Jul} 7$ \\
\hline M71 & $g^{\prime}$ & 5 & 30 & 1 ". 3 & $2015 \mathrm{Jul} 7$ \\
\hline
\end{tabular}

USNO-B1.0 catalog (Monet et al. 2003), and crowded field PSF-fitting photometry was performed using IRAF/DAOPHOT (Stetson 1987), following the procedures outlined by Davis et al. (1994). Finally, to place the $g^{\prime}$ data on an absolute scale, we used the astrometric coordinates of each star to identify its counterpart in the SDSS catalog (An et al. 2008). After excluding known variable stars (Samus et al. 2009) and objects within $\sim 1$ effective radius of the cluster center (which have poor SDSS photometry due to crowding), we computed the magnitude difference between our instrumental $g^{\prime}$ magnitudes and those found by SDSS. For red giants measured from our shallower images, we found that crowding may contribute to increased photometric errors within $20^{\prime \prime}$ of the cluster center, and we therefore excluded stars within that radius in M3, M5, and M13 from our analysis. M71 is sufficiently sparse that it requires no such exclusion of central stars.

Figure 1 displays the residuals between our measurements and that of the SDSS catalog. The slight slope in the stellar locii, $\sim 0.01 \Delta g^{\prime} / g_{\text {inst }}^{\prime}$, is due to the presence of a small colorterm between the SDSS and WIYN $g^{\prime}$ systems and is of no significance for our analysis, as it is removed when we fit a fiducial RGB. Note that in the magnitude range of our program's RGB stars (the faint end is identified by the dotted lines in the figure), the scatter between our $g^{\prime}$ measurements and those of SDSS is $\sim 4 \%$, independent of stellar luminosity. Because the photometric error associated with SDSS $g^{\prime}$ photometry is $\sim 2 \%$, this suggests that the internal uncertainty associated with our $g^{\prime}$ magnitudes is $\sim 3 \%$. This number is considerably larger than the internal errors reported by the DAOPHOT allstar algorithm.

The zero point of our CN-band photometry was set to match that of the SDSS $u^{\prime}$ system. Specifically, we took the SDSS 


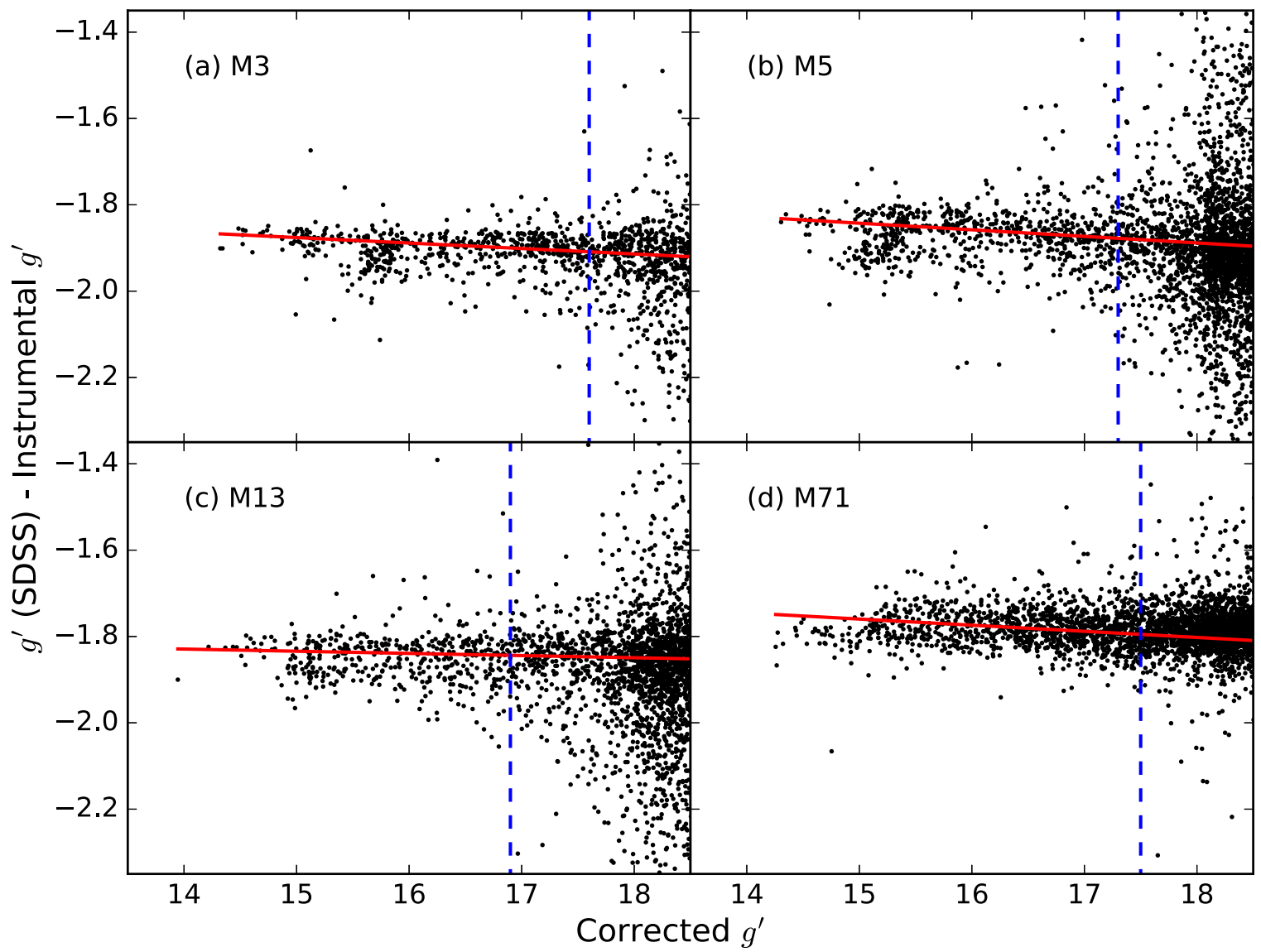

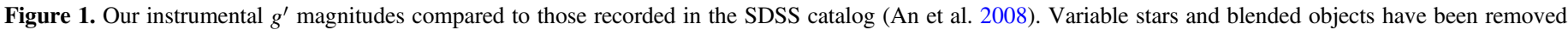

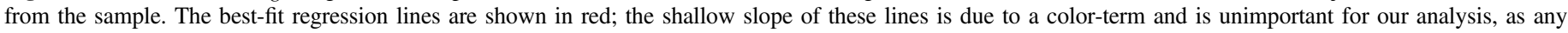

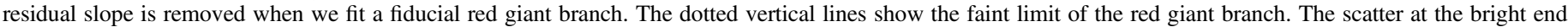
suggests that the one sigma photometric errors for RGB stars is $\sim 3 \%$.

$u^{\prime}-g^{\prime}$ color of each GC's main-sequence turnoff (given in Table 1) and shifted our $\mathrm{CN}$ magnitudes so that the $\mathrm{CN}-g^{\prime}$ color of the cluster's main-sequence turnoff matched this color. Figure 2 displays the resultant CMDs. Note that because our analysis utilizes differential colors relative to a fiducial giant branch, this zero point is arbitrary.

\section{Spectroscopic versus Photometric Abundances}

The goal of our experiment is to measure the sensitivity of RGB CN $-g^{\prime}$ colors to changes in the stellar population. This can most easily be done by using RGB stars whose chemical abundances have been determined spectroscopically. In particular, the proton-capture element sodium, when normalized to iron, is an excellent indicator of chemical enrichment due to proton-capture nucleosynthesis.

Five samples of $[\mathrm{Na} / \mathrm{Fe}]$ abundances are suitable for our analysis. Our primary source of abundance data is the WIYNHydra based measurements of Johnson et al. (2005), who determined $[\mathrm{Na} / \mathrm{Fe}]$ values for 76 stars in $\mathrm{M} 3$ and 112 stars in M13 to a precision of 0.05 dex. For the GC M71, the $[\mathrm{Na} / \mathrm{Fe}]$ measurements come from two sources: the 33 WIYN-Hydra observations of Cordero (2014), which have an abundance precision of 0.05 dex, and the 25 stars measured by Ramírez \& Cohen (2002) using the Keck Telescope's High Resolution Echelle Spectrograph (0.08 dex precision). An abundance comparison of the six stars common to both studies indicates the two samples are statistically consistent and their error estimates are reasonable. The $[\mathrm{Na} / \mathrm{Fe}]$ measurements for the cluster M5 are also taken from two sources. Cordero (2014) measured $[\mathrm{Na} / \mathrm{Fe}]$ values for 61 stars in M5, each with a precision of 0.05 dex, and Carretta et al. (2009a) and Carretta et al. (2009b) measured 122 stars using the ESO UVES and GIRAFFE fiber-fed spectrographs with typical uncertainties of 0.05 dex. The abundance measurements for 16 stars that appear in both samples are in good agreement. All $[\mathrm{Na} / \mathrm{Fe}]$ abundances assume local thermal equilibrium (LTE) conditions.

In total, the sample of stars with known $[\mathrm{Na} / \mathrm{Fe}]$ abundances consists of 407 objects. The largest $[\mathrm{Na} / \mathrm{Fe}]$ spread is displayed by the stars in $\mathrm{M} 13$, where $\Delta[\mathrm{Na} / \mathrm{Fe}]$ is $1.37 \mathrm{dex}$, while the smallest spread is in $\mathrm{M} 71$, where $\Delta[\mathrm{Na} / \mathrm{Fe}]=0.92$ dex. M3 and M5 are intermediate cases, with $\Delta[\mathrm{Na} / \mathrm{Fe}]=1.00$ dex. Carretta et al. (2010) found that the extent of the Na-O anticorrelation, as well as the range of the $[\mathrm{Na} / \mathrm{Fe}]$ abundances, among stars in a cluster correlate with cluster mass, metallicity, and Galactic origin (inner/outer halo versus disk).

To examine the sensitivity of the $\mathrm{CN}-g^{\prime}$ color system to stellar population, we created a fiducial RGB for each cluster. We selected those RGB stars within the magnitude range of the $[\mathrm{Na} / \mathrm{Fe}]$ spectroscopy, and fit their locus with a third order polynomial (see Figure 3) by minimizing the scatter in color using ordinary least squares (OLSs). We then considered each star with a spectroscopic $[\mathrm{Na} / \mathrm{Fe}]$ determination, and correlated its distance from the fiducial line, $\Delta\left(\mathrm{CN}-g^{\prime}\right)$ (measured color minus fitted color), with its spectroscopically determined value 


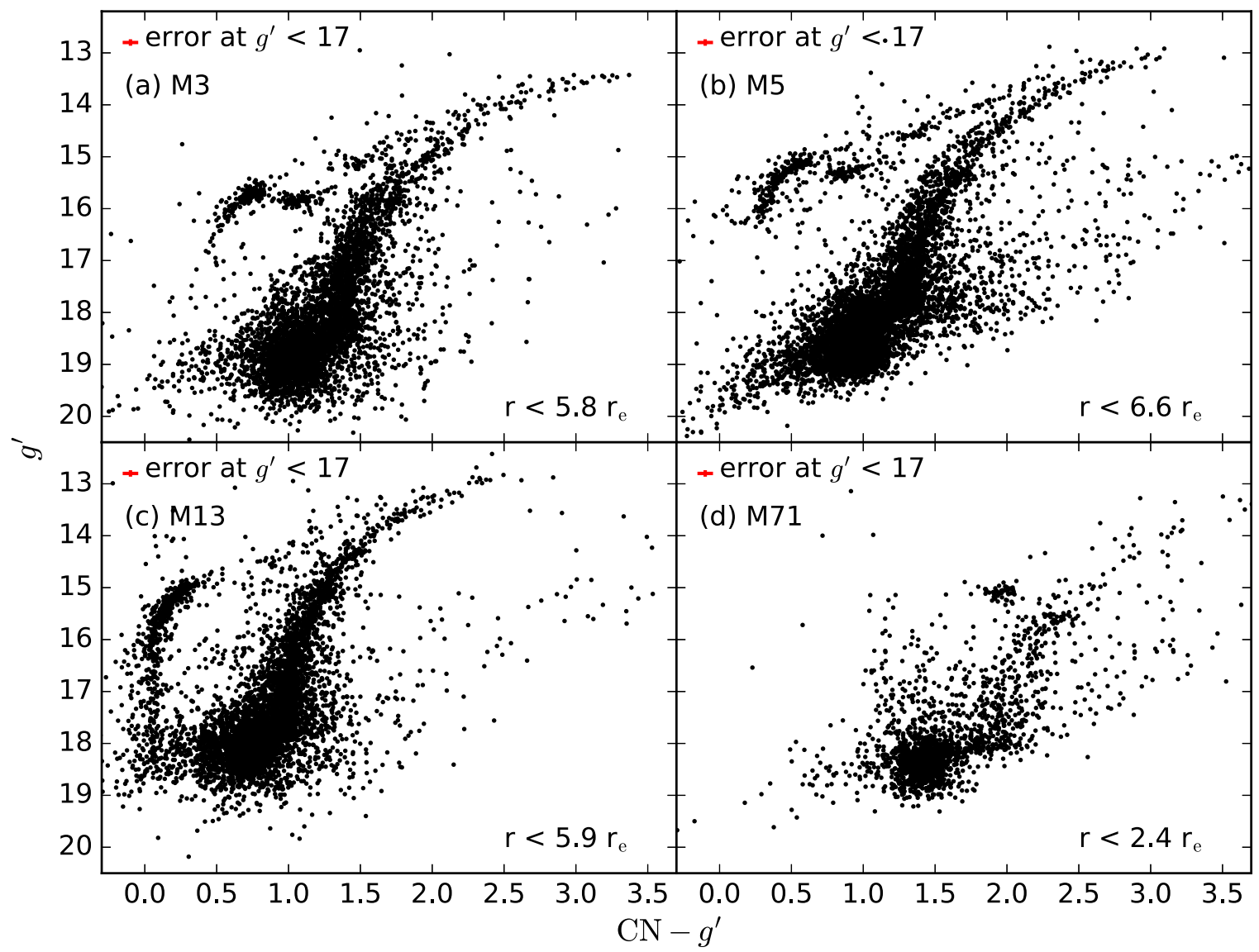

Figure 2. Color-magnitude diagrams for the four GCs, with $g^{\prime}$ magnitudes plotted against $\mathrm{CN}-g^{\prime}$ colors. The $\mathrm{CN}$ zero point is based on the SDSS $u^{\prime}$ magnitude at the main-sequence turnoff. Variable stars have been removed from the figure. The typical one sigma photometric error of giant branch stars is shown via the red cross in the upper right hand corner of each panel. Note that the width of the giant branch is much larger than that predicted from the photometric errors.

of $[\mathrm{Na} / \mathrm{Fe}]$. These comparisons are shown in Figure 4 and summarized in Table 3.

As expected, Figure 4 shows a correlation between $\mathrm{CN}-g^{\prime}$ and $[\mathrm{Na} / \mathrm{Fe}]$ index: higher metallicity stars have deeper $\mathrm{CN}$ absorption troughs, hence fainter $\mathrm{CN}$ magnitudes and redder $\mathrm{CN}-g^{\prime}$ colors. For the three intermediate-metallicity, low-reddening systems, the significance of this correlation is much greater than $99 \%$ (according to the Pearson correlation coefficient), and the slopes of the relation are similar. In addition, the scatter of the data around the correlation is consistent with the reported uncertainties in $[\mathrm{Na} / \mathrm{Fe}]$ and $\Delta\left(\mathrm{CN}-g^{\prime}\right)$. For M71, the correlation between color and $[\mathrm{Na} / \mathrm{Fe}]$ abundance is less clear, possibly due to its higher overall metallicity and saturation of the CN-band (Suntzeff 1981; Langer 1985). Our result for M71 is consistent with that of Smith (2015b), who measured a moderately strong correlation between spectroscopic $\mathrm{CN}-$ band strength and $[\mathrm{Na} / \mathrm{Fe}]$ abundance in the cluster's RGB stars. For M5, we see a more robust correlation between $\mathrm{CN}$ strength and $[\mathrm{Na} / \mathrm{Fe}]$ than was reported by Smith et al. (2013), likely due to our larger sample.

To quantitatively determine the dependence of $\mathrm{CN}-g^{\prime}$ color on $[\mathrm{Na} / \mathrm{Fe}]$, we followed the recommendation of Feigelson \& Babu (1992) and used the OLSs bisector method to compute the slope of a $\Delta\left(\mathrm{CN}-g^{\prime}\right)$ versus $[\mathrm{Na} / \mathrm{Fe}]$ line. This method consists of computing two OLS solutions, in turn treating each variable as independent, and selecting the line that bisects these solutions. The best-fit slope and its uncertainty is then found via a bootstrap analysis, which in our case, used 10,000 realizations.
The corresponding intercept is found by minimizing the scatter in color. The best-fit line, shown in red in Figure 4, uses the slope and intercept given in Table 3 and has the form

$$
[\mathrm{Na} / \mathrm{Fe}]=(\text { slope }) \times \Delta\left(\mathrm{CN}-g^{\prime}\right)+(\text { intercept }) .
$$

\section{The Distribution of Abundances}

The width of the RGB (almost $0.6 \mathrm{mag}$ ) in the $\mathrm{CN}-g^{\prime}$ color in each cluster shown in Figure 3 is broader than that expected from photometric uncertainties $(0.03 \mathrm{mag})$ and demonstrates that the $\mathrm{CN}-g^{\prime}$ color system is sensitive to stellar abundance variations. This finding was confirmed from photometry of artificial stars added along each cluster's fiducial giant branch in our $g^{\prime}$ and $\mathrm{CN}$ image frames. While there are other ways to gain this increased sensitivity, such as through the use of Strömgren photometry (e.g., Calamida et al. 2007), Washington system photometry (e.g., Cummings et al. 2014), broadband ultraviolet colors (Milone et al. 2017), the CTIO Ca filter ( $\mathrm{Lim}$ et al. 2015), and measurements of calcium H \& K (e.g., Roh et al. 2011), the $\mathrm{CN}$ measurements offer a fairly direct and efficient path to probe the range of light-element abundances in GCs.

The $\mathrm{CN}-g^{\prime}$ photometry also allows us to study the radial dependence of abundance variations within each cluster. Our results are illustrated in Figure 5, where we have divided the RGB stars of each system into three radial bins, each containing an equal number of stars. The top axes of the 


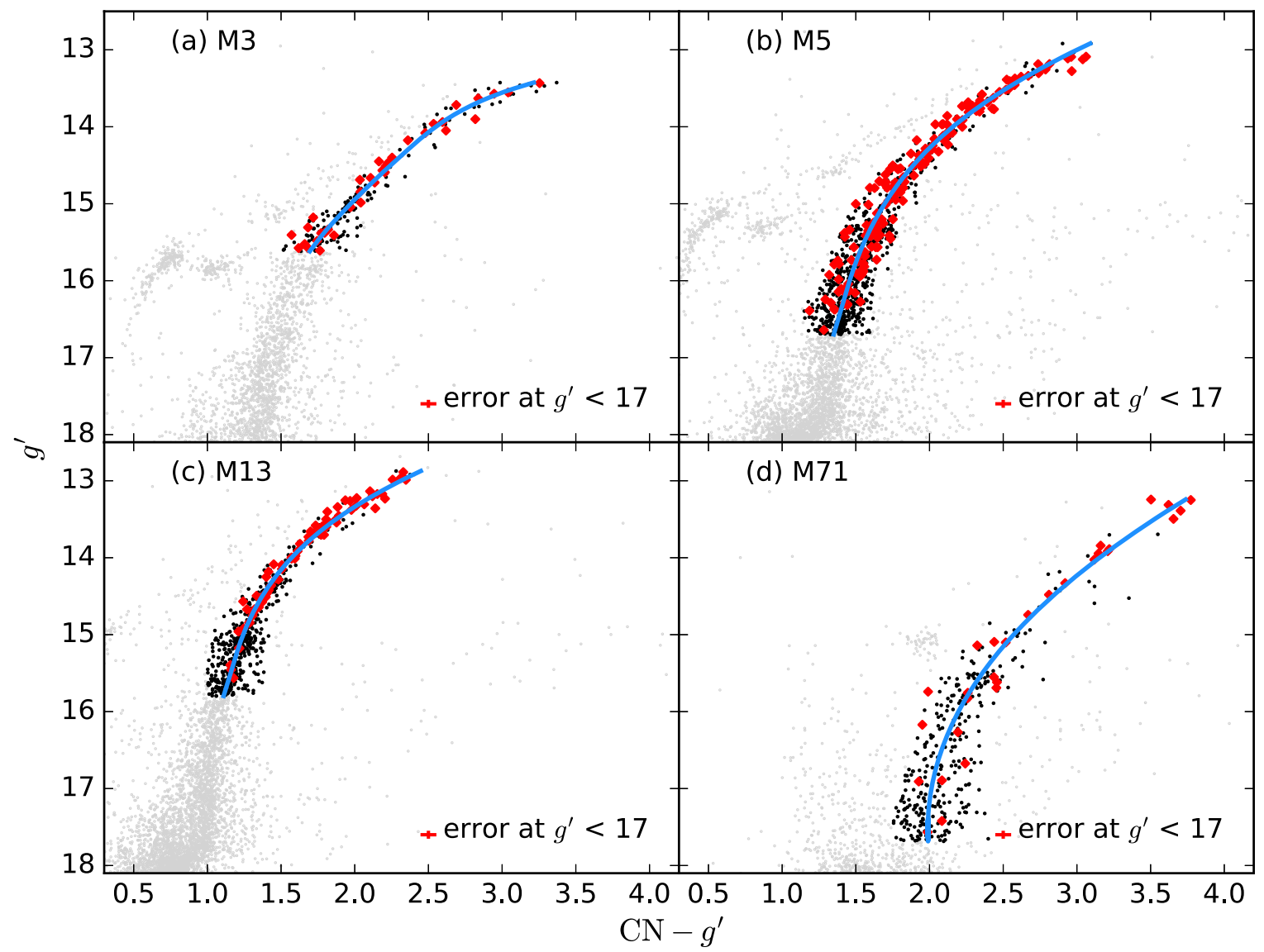

Figure 3. The red giant branch of the CMD of each cluster. Our fiducial mean RGB track is displayed in blue. The stars that were used to produce this track are shown in black, while stars not used in the fit are displayed in gray. Stars with known $[\mathrm{Na} / \mathrm{Fe}]$ abundances are identified with red diamonds.

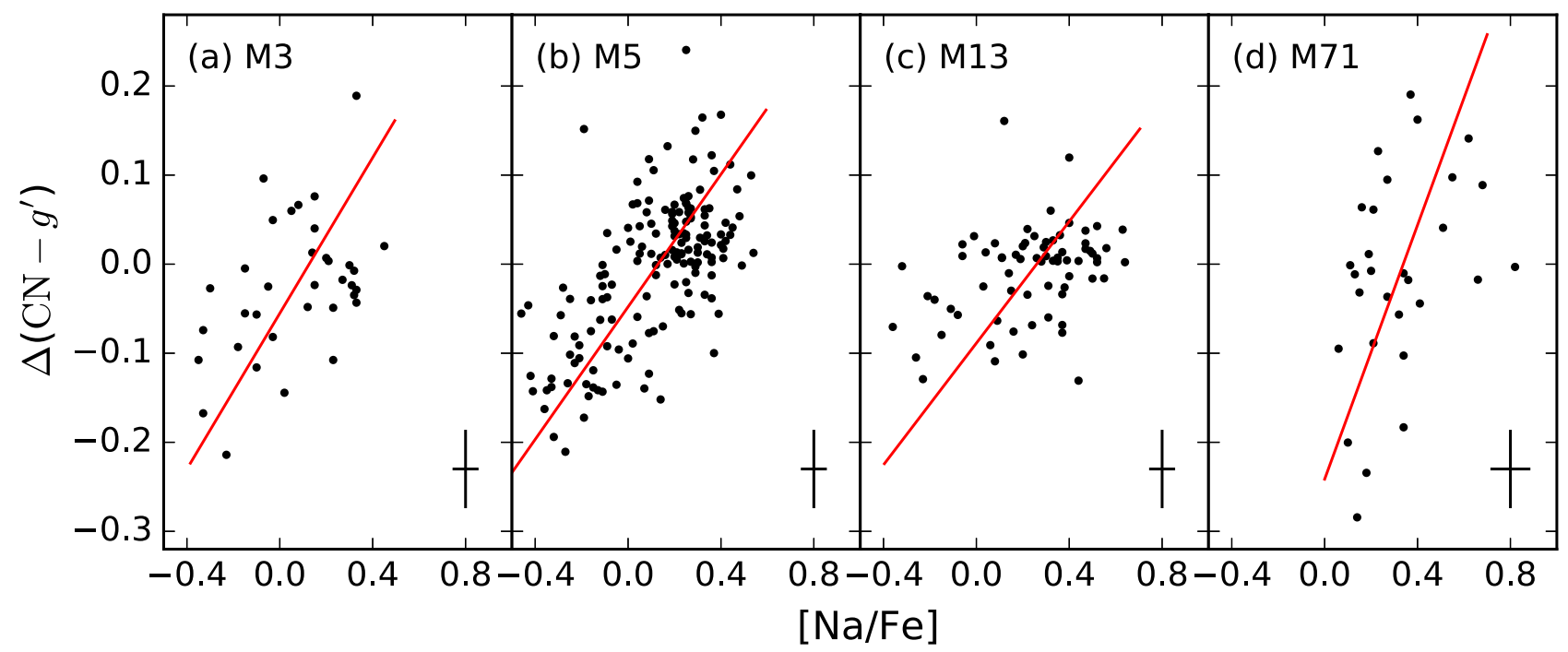

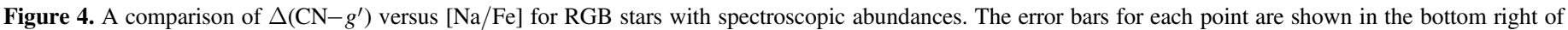

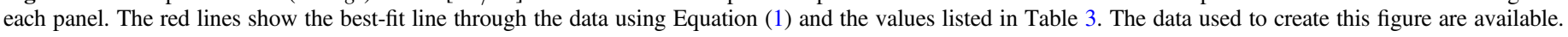

plots translates these values into $[\mathrm{Na} / \mathrm{Fe}]$ using Equation (1) and assumes that $\Delta\left(\mathrm{CN}-g^{\prime}\right)$ perfectly traces $[\mathrm{Na} / \mathrm{Fe}]$ abundance. Even a cursory examination of the data suggests that some degree of population segregation exists in most clusters. In particular, a Kolmogorov-Smirnov (K-S) comparison between the innermost and outermost subsets demonstrates that the $\mathrm{CN}$ spread in the inner parts of M13,
M3, and M5 is much wider than that seen in the systems' outer regions. This result, which is significant at more than 95\% confidence (see Table 5), agrees with the conclusions of previous studies (e.g., Carretta et al. 2010; Johnson \& Pilachowski 2012; Cordero 2014), at least in the dynamically young clusters. There is also a notable deficit of stars with strong CN-bands in the outer regions of M3 and M13, 
Table 3

Summary of the Correlation Between $\Delta\left(\mathrm{CN}-g^{\prime}\right)$ and $[\mathrm{Na} / \mathrm{Fe}]$

\begin{tabular}{|c|c|c|c|c|c|c|}
\hline Cluster & $\begin{array}{c}\text { Number of } \\
\text { Stars }\end{array}$ & $\begin{array}{l}\text { Magnitude } \\
\text { Limit }\end{array}$ & $\begin{array}{l}\text { Correlation } \\
\text { Coefficient }\end{array}$ & $\begin{array}{c}p \text {-value } \\
\text { two-tailed }\end{array}$ & $\begin{array}{c}\text { Slope } \\
{[\mathrm{dex} / \mathrm{mag}]}\end{array}$ & $\begin{array}{c}\text { Intercept at } \\
\Delta\left(\mathrm{CN}-g^{\prime}\right)=0\end{array}$ \\
\hline M3 & 35 & 15.6 & 0.46 & 0.0053 & $2.29 \pm 0.50$ & 0.13 \\
\hline M5 & 159 & 16.7 & 0.63 & $8 \times 10^{-19}$ & $2.69 \pm 0.18$ & 0.13 \\
\hline M13 & 70 & 15.8 & 0.36 & 0.0023 & $2.93 \pm 0.91$ & 0.26 \\
\hline M71 & 29 & 17.7 & 0.39 & 0.0355 & $1.40 \pm 0.23$ & 0.34 \\
\hline
\end{tabular}

$[\mathrm{Na} / \mathrm{Fe}]$
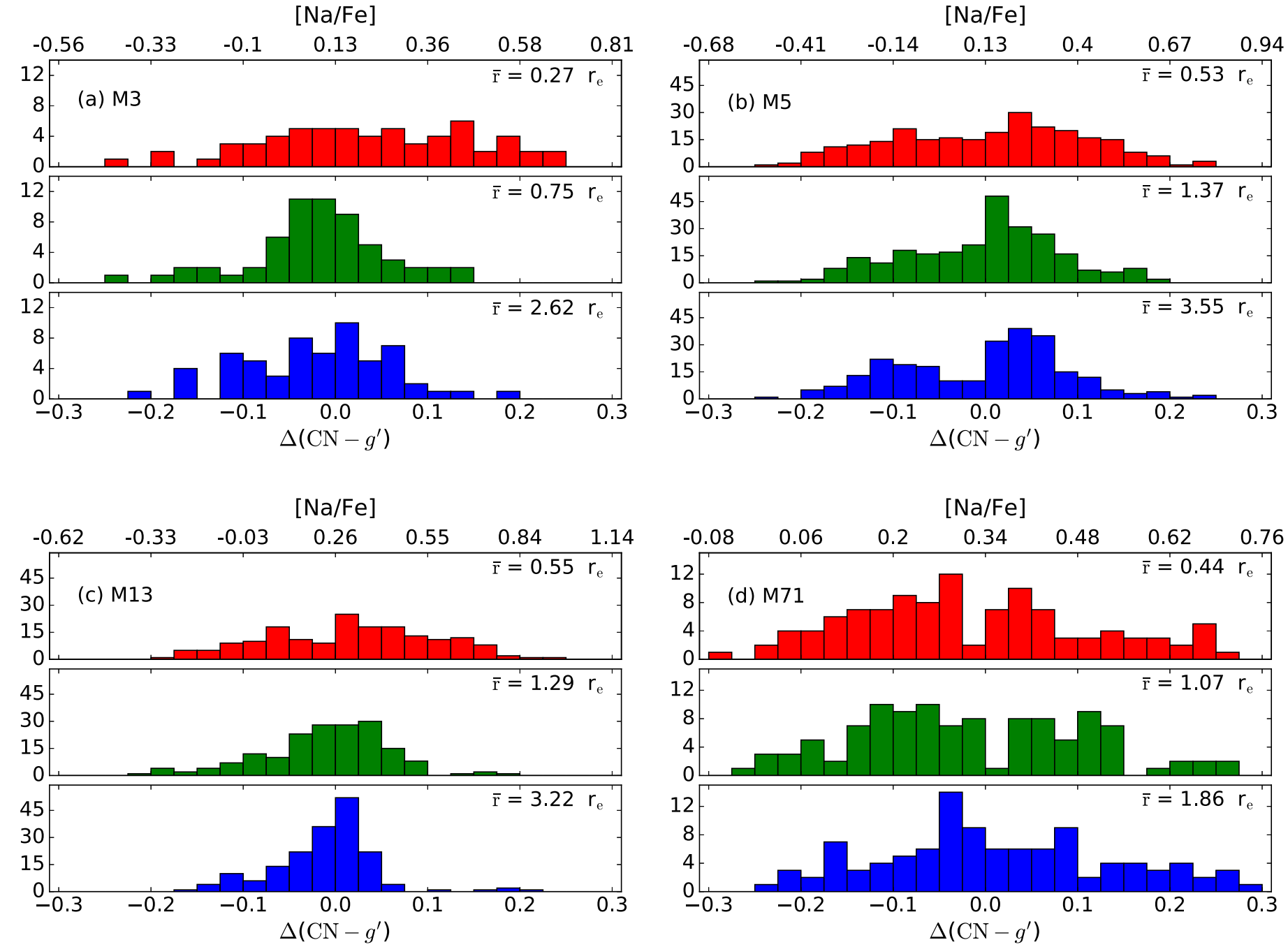

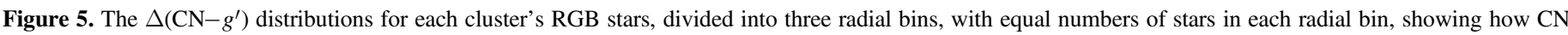

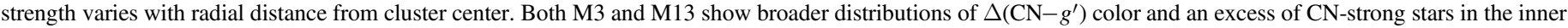

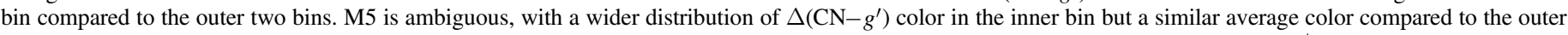

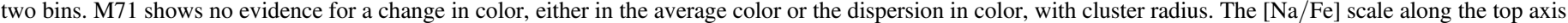
of each plot is calibrated for each cluster using Equation (1) and the values in Table 3.

Table 4

The Radii Dividing the RGB Sample for Each Cluster into Three Bins Containing an Equal Number of Stars

\begin{tabular}{lccc}
\hline \hline Cluster & $\begin{array}{c}\text { Outer Radius of } \\
\text { Inner Bin }\left(r_{e}\right)\end{array}$ & $\begin{array}{c}\text { Inner Radius of } \\
\text { Outer Bin }\left(r_{e}\right)\end{array}$ & $\begin{array}{c}\text { Total stars in } \\
\text { RGB sample }\end{array}$ \\
\hline M3 & 0.45 & 1.18 & 181 \\
M5 & 0.87 & 2.04 & 762 \\
M13 & 0.87 & 1.83 & 529 \\
M71 & 0.75 & 1.38 & 331 \\
\hline
\end{tabular}

suggesting that the $\mathrm{CN}$-enriched stars, which may be associated with the cluster's SG, are more centrally concentrated. The dependence is much weaker in M5, as the average $\Delta\left(\mathrm{CN}-g^{\prime}\right)$ values are roughly equal in the three radial bins. M71 displays no evidence for a population gradient, though given the cluster's short dynamical timescale, this result is not surprising. The lack of a population gradient in M71 is also consistent with the $[\mathrm{Na} / \mathrm{Fe}]$ analysis of Cordero (2014), who found a high degree of spatial mixing between the primordial and enriched populations of the cluster. 
Table 5

Results of a Two-sample K-S Test of the $\Delta\left(\mathrm{CN}-g^{\prime}\right)$ Distributions between the Inner and Outer Third of Cluster Stars, and the Fraction of Second Generation (SG) Stars in Each Radial Bin

\begin{tabular}{lccccc}
\hline \hline Cluster & $\begin{array}{c}p \text {-value } \\
\text { (two-tailed) }\end{array}$ & $\begin{array}{c}\text { Mean } \\
\text { Inner }\end{array}$ & $\begin{array}{c}(\text { St.Dev }) \\
\text { Middle }\end{array}$ & $\begin{array}{c}\Delta\left(\mathrm{CN}-g^{\prime}\right) \\
\text { Outer }\end{array}$ & $\begin{array}{c}\text { SG stars }(\%) \\
\text { Inner/Middle/Outer }\end{array}$ \\
\hline M3 & 0.0040 & $0.035(0.11)$ & $-0.017(0.07)$ & $-0.020(0.08)$ \\
M5 & 0.044 & $0.004(0.11)$ & $-0.002(0.08)$ & $-0.002(0.09)$ & $61 / 38 / 45$ \\
M13 & $4.39 \times 10^{-11}$ & $0.017(0.09)$ & $-0.010(0.07)$ & $-0.011(0.06)$ & $65 / 57 / 58$ \\
M71 & 0.069 & $-0.015(0.13)$ & $-0.020(0.12)$ & $0.030(0.15)$ & $44 / 48 / 47$ \\
\hline
\end{tabular}

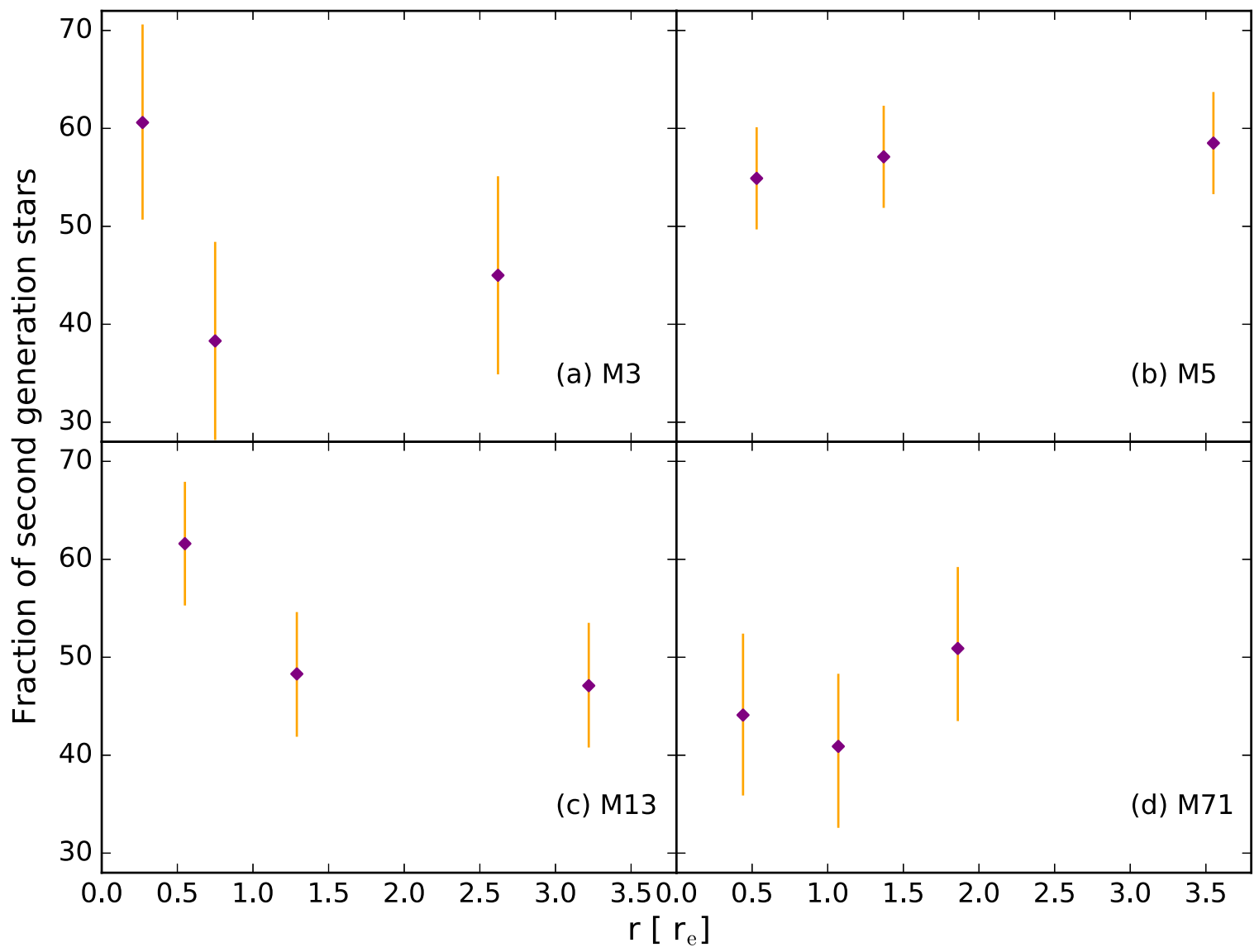

Figure 6. The population ratio (second generation stars relative to total) for the three radial bins described in Figure 5 and Table 4 . Second generation stars are identified as those lying redward of the fiducial RGB shown in Figure 3 (i.e., stars with strong CN-bands). The ratios are found via a bootstrap analysis using 10,000 samples, and the error bars reflect $90 \%$ confidence intervals. M3 and M13 show evidence for a change in the population ratio with radius, with second generation stars more centrally concentrated than the primordial population. The other two clusters show no such trend.

To further demonstrate population segregation in the clusters, we divided each cluster's RGB stars into two samples: the CN-weak stars, which lie blueward of the mean RGB displayed in Figure 3 and are associated with the cluster's first generation (FG), and $\mathrm{CN}$-strong (second generation) stars, which fall redward of this fiducial curve. We examined the radial distribution of these samples by computing the population ratio (the fraction of SG stars) for each of the three radial bins described above and via the empirical cumulative distribution function (ECDF). These distributions are shown in Figure 6 and Figure 7, respectively. The population ratio and corresponding uncertainty (90\% confidence intervals) are determined using a bootstrap analysis (10,000 samples). The figures demonstrate that M3 and M13 have higher percentages of SG, CN-strong stars in their inner regions than in their outer parts, confirming our measurement of the clusters' population gradient. There is no compelling evidence for this segregation in M5: the $\mathrm{CN}$-strong and $\mathrm{CN}$-weak stars have statistically similar distributions. Cordero (2014) also found no radial dependence in the $[\mathrm{Na} / \mathrm{Fe}]$ abundances in $\mathrm{M} 5$, in contrast to the gradient detected by Lardo et al. (2011). In M71, the distributions of primordial and enriched stars appear to be identical, as is expected for a well-mixed cluster.

\section{Integrated Light}

The next step toward understanding the stellar populations of GCs is to study these systems in other galaxies, where the conditions of formation may have been quite different from that which occurred in the Milky Way. This presents a problem, however, as at distances larger than a few hundred kiloparsecs, spectroscopic measurements of individual stars become prohibitively difficult. However, the sensitivity of the 


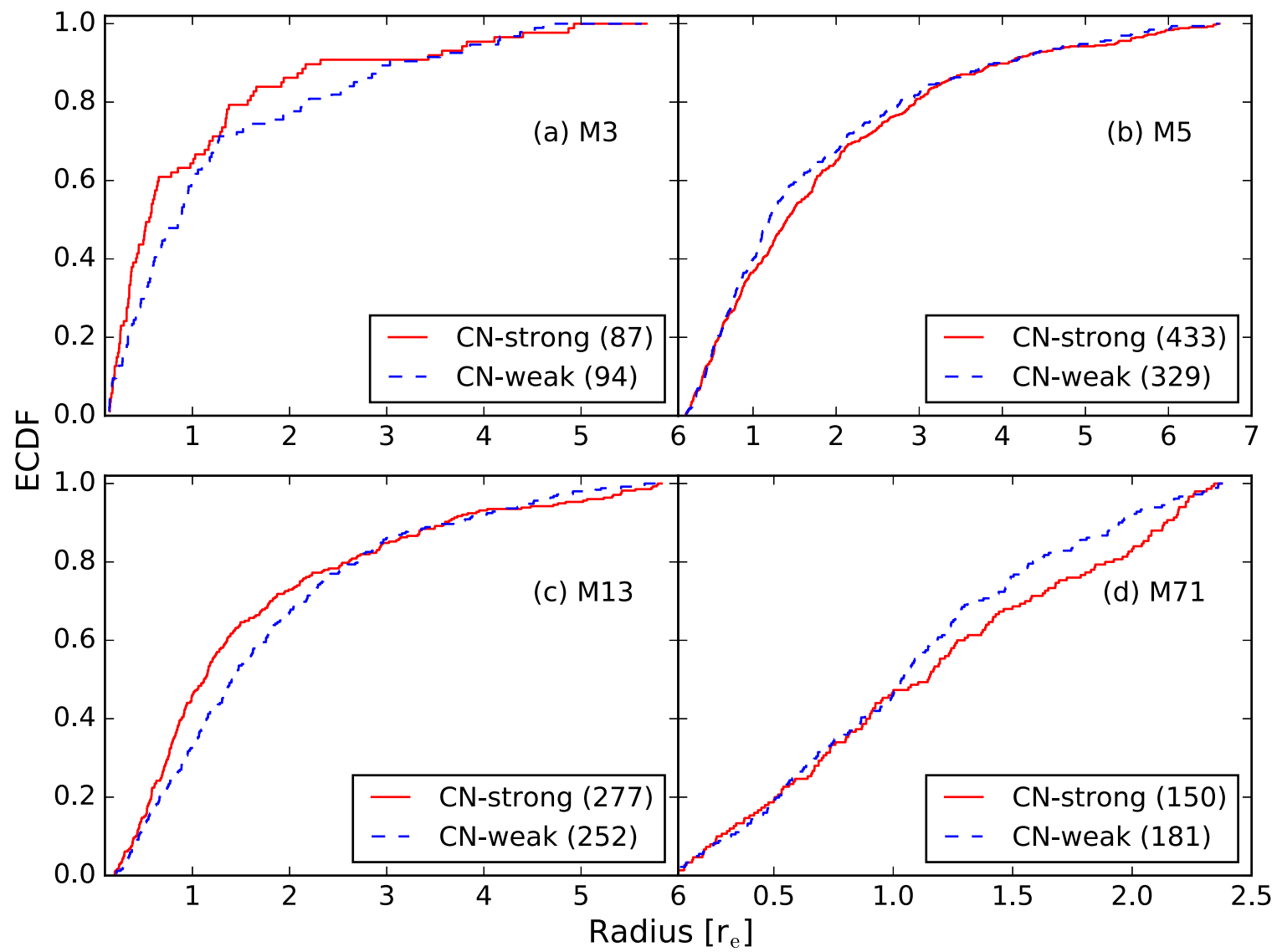

Figure 7. The empirical cumulative distribution functions for $\mathrm{CN}$-strong stars (red solid line) and $\mathrm{CN}$-weak stars (blue dashed line) in each cluster, where the two groups are separated by the fiducial RGB curves shown in Figure 3. The number of stars in each sample are given in the plot legends. The CN-strong stars, which are likely second generation objects, appear to be more centrally concentrated in M3 and M13. The CN-strong and CN-weak stars in M5 have statistically similar distributions. The dynamically relaxed cluster M71 shows no evidence for radial segregation.

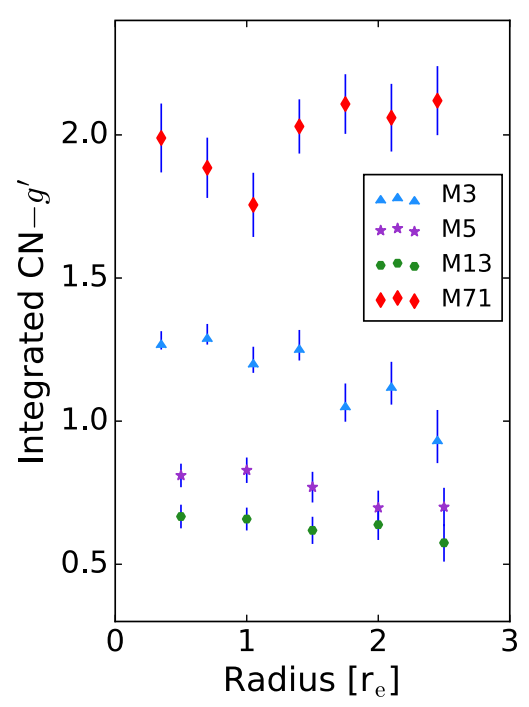

Figure 8. Integrated $\mathrm{CN}-g^{\prime}$ colors in different annuli are shown for each cluster. The CN zero point is based on the SDSS $u^{\prime}$ magnitude at the mainsequence turnoff. Errors on the points are estimated from the $g^{\prime}$ luminosity contained in each region using Equation (2). Note that the photometric error bars on each color measurement are smaller than the points. In $\mathrm{M} 3$, the $\mathrm{CN}-g^{\prime}$ color becomes bluer at larger radii, signifying fewer second generation stars in the outer regions. (A greater number of $\mathrm{CN}$-strong stars leads to redder colors, because the enriched stars absorb more light in the blue $\mathrm{CN}$-band). Within our uncertainties, the integrated colors of M5, M13, and M71 are independent of distance from cluster center. Our data are limited to 2.5 effective radii in order to avoid possible systematic errors associated with low surface brightness photometry.
$\mathrm{CN}-g^{\prime}$ color index gives us a tool with which to explore the chemical homogeneity of clusters, even at large distances.

Figure 8 mimics what might be recorded if the four GCs were at the distance of M31 by displaying their integrated $\mathrm{CN}-g^{\prime}$ colors as a function of cluster radius. From the figure, it is clear that the radial $\mathrm{CN}-g^{\prime}$ color gradient, which is seen in the individual RGB star abundances of the dynamically young system M3, is easily detected. For M5 and M13, the integrated $\mathrm{CN}-g^{\prime}$ colors remain constant to $\sim 1.5$ effective radii but show evidence for a possible decline beyond this radius. Conversely, M71, which is dynamically old and well mixed, shows no gradient either in the individual RGB abundances or its integrated color. This demonstrates the feasibility of detecting multiple stellar populations within a GC, even if that cluster is too far away for its stars to be resolved. Note that we limit the radial extent of our integrated color analysis to $\sim 2.5$ effective radii to avoid systematic offsets due to possible background contamination at low surface brightness.

A reasonable concern with this integrated light approach is its sensitivity to stochastic processes associated with the distribution of stars. The integrated color of a stellar population depends on the number of bright objects present in the aperture. For old systems, such as GCs, this primarily means bright RGB and AGB stars, though under some conditions, post-AGB stars and bright field objects may also be important. In lowluminosity systems (i.e., all GCs) the number of these bright stars may be small, leading to color fluctuations, even in homogeneous simple stellar populations. Under the right 


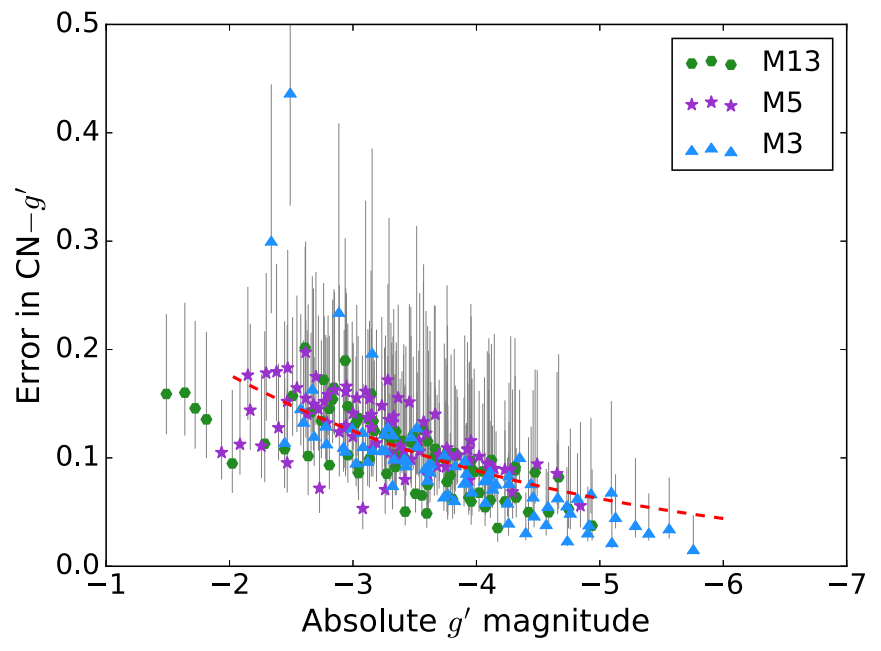

Figure 9. The stochasticity in integrated $\mathrm{CN}-g^{\prime}$ colors as a function of absolute $g^{\prime}$ magnitude. The $\mathrm{CN}$ zero point is based on the SDSS $u^{\prime}$ magnitude at the main-sequence turnoff. The data were generated by dividing a given radial bin into several azimuthal subsections and measuring the color dispersion as a function of luminosity in the annulus. M71 has been omitted from the comparison, due to contamination from foreground field stars. Gray lines show the $90 \%$ confidence intervals in the measured color dispersion. The red line gives the best fit in the form of an exponential decay. This plot shows that for all systems with an integrated absolute $g^{\prime}$ magnitude brighter than $g^{\prime} \sim-5$, the uncertainty in the measurement of integrated $\mathrm{CN}-g^{\prime}$ colors due to stellar stochasticity is quite small.

circumstances, these stochastic effects may mimic the signature of a change in stellar population.

To suppress the effect of stellar stochasticity in the gradient measurements of Figure 8, the $\mathrm{CN}-g^{\prime}$ color of each radial bin was derived from the median of 19 different azimuthal slices within the cluster. Unfortunately, when measuring clusters outside of the Milky Way, it may not always be possible to use such fine subdivisions. Thus, to understand quantitatively the effect that stochasticity has on the colors, we excluded M71 from the analysis (due to the high density of field stars at its location) and used our data on the remaining three intermediate-metallicity clusters to compare sets of color estimates made at fixed radii but at different azimuthal position angles. Specifically, we divided each radial annulus into several azimuthal bins and measured the color in each bin. Because we expect a cluster's stellar population to be constant within a given radial annulus, the changes in color as a function of azimuth should provide a measure of the scatter introduced by stellar stochasticity.

Figure 9 displays the results of this analysis, along with the $90 \%$ confidence intervals on each dispersion measurement. It is clear from the figure that for sample regions of a cluster with an integrated absolute $g^{\prime}$ magnitude brighter than $g^{\prime} \sim-5$, the uncertainty introduced by measuring a finite number of stars is small. If we fit an exponential decay model to the color error shown in Figure 9, then

$$
\sigma\left(\mathrm{CN}-g^{\prime}\right) \sim 0.35 \times 0.83^{-1.84 \times g^{\prime}} .
$$

This is shown as the red dashed line in the plot. Of course, Figure 9 represents a lower limit to the effect of stellar stochasticity; it is possible that when observing distant clusters, the survey will include some rare, bright sources that are not present in the finite populations of M3, M5, and M13. Nevertheless, the plot does demonstrate that under most circumstances, the errors associated with stellar evolution are minimal, and in our specific case, the color gradients displayed in Figure 8 are real.

\section{Discussion}

The correlation of the $\Delta\left(\mathrm{CN}-g^{\prime}\right)$ color with published $\mathrm{Na}$ abundance, as shown in Section 4, establishes the utility of the this color index to distinguish and track multiple populations in GCs. In Section 5, artificial star tests confirm that all four of the clusters observed show a wider distribution of $\Delta\left(\mathrm{CN}-g^{\prime}\right)$ color than would be expected from observational uncertainties, supporting the evidence of other photometric and spectroscopic studies for the presence of multiple stellar populations in GCs.

The $\mathrm{CN}-\mathrm{g}^{\prime}$ color index provides us with an opportunity to efficiently characterize the stellar population gradients in GCs. As described in Section 5, we used individual stars to examine histograms of $\Delta\left(\mathrm{CN}-g^{\prime}\right)$ in three radial bins and to compare the distribution of $\mathrm{CN}$-weak and $\mathrm{CN}$-strong stars in the inner and outer regions of the clusters. We also computed the population ratio (the fraction of SG stars) for each of the three bins and compared the cumulative distribution functions for $\mathrm{CN}$-strong and $\mathrm{CN}$-weak stars as a function of distance from the clusters' centers. Finally, in Section 6, we look for gradients in the $\mathrm{CN}-g^{\prime}$ colors of our clusters using the systems' integrated light that could be associated with changes in the fraction of $\mathrm{CN}$-strong and $\mathrm{CN}$-weak stars with distance from cluster center. We now examine the results for each cluster in the context of dynamical evolution and compare our measurements with previous work.

M3, with a present-day median relaxation time of $6.2 \mathrm{Gyr}$ (Harris 2010) and an age of 11.75 Gyr (VandenBerg et al. 2013), is, dynamically speaking, the youngest of the GCs in our sample, as well as one of the youngest clusters of the Milky Way. Histograms of the individual stars' $\Delta\left(\mathrm{CN}-g^{\prime}\right)$ index, divided equally into three radial bins (Figure 5), show an excess of $\mathrm{CN}$-strong stars in the inner bin, within $0.45 r_{e}$ of the cluster center.

This result is corroborated by Figure 6, which shows an SG stellar population that is more centrally concentrated than the primordial population. Likewise, the ECDF (Figure 7) for M3 stars shows that $\mathrm{CN}$-strong stars are more centrally concentrated than $\mathrm{CN}$-weak stars. In integrated light (Figure 8), we find a clear gradient in the $\mathrm{CN}-g^{\prime}$ color corresponding to a decrease in $\mathrm{CN}$ strength as one moves outwards from one to three effective radii.

Studies of stellar populations in M3 by Johnson et al. (2005), Lardo et al. (2011), and Massari et al. (2016) provide evidence of significant differences in the central concentration of its stellar populations. As discussed in Section 4, Johnson et al. (2005) determined $\mathrm{Na}$ abundances for several dozen stars in M3. Dividing the sample in half using their sodium abundances, we obtain a cumulative distribution function from the sodium abundance data that is qualitatively similar to the cumulative distribution function determined from our $\Delta\left(\mathrm{CN}-g^{\prime}\right)$ photometry; both show a clear central concentration of Na-rich stars compared to Na-poor stars. We note that the stars analyzed by Johnson et al. (2005) lie mostly outside of a radial distance of 0.5 effective radii from the center of $\mathrm{M} 3$, and their sample extends beyond 8 effective radii.

Massari et al. (2016) used Strömgren photometry, specifically the $c_{y}$ color, to separate the stellar populations of M3. This index, defined by Yong et al. (2008), is sensitive to CN line 
strength but not temperature. M3's two populations are separated by about $0.05 \mathrm{mag}$ in $c_{y}$ along the cluster's lower RGB. Again, their cumulative distribution function for M3 is qualitatively similar to what we obtain using $\Delta\left(\mathrm{CN}-g^{\prime}\right)$ photometry.

Lardo et al. (2011) examined the radial distributions of red giant stars in several GCs using $\left(u^{\prime}-g^{\prime}\right)$ colors from SDSS (Abazajian et al. 2009 and references therein), as reanalyzed by An et al. (2008). Again, our cumulative distribution function for M3 is in qualitative agreement with that of Lardo et al. (2011), thereby confirming gradients in the population ratio in this cluster.

The presence of a population gradient in M3 is consistent with predictions. Vesperini et al. (2013) demonstrate that differences in the initial central concentrations of M3's stellar populations will survive at least until the present day. For their least-centrally concentrated $\mathrm{r} 2 \mathrm{p} 5$ model, with an initial ratio of the half-mass radius of FG stars to the half-mass radius of SG stars of $R_{h, \mathrm{FG}} / R_{h, \mathrm{SG}}=2.5$, the initial central concentrations remain near the original value for several relaxation times, and then begin to drop gradually over many relaxation times. For more highly concentrated initial conditions for SG stars, the central concentration difference is erased more quickly. In all of their simulations, however, the higher central concentration of SG stars persists well beyond the dynamical age of M3. As clusters age, SG stars migrate outwards and the steepness of the population ratio gradient declines. By two relaxation times, the population ratio is constant out to roughly 0.5 half-mass radii and declines beyond that point. This general behavior is consistent with the decline in M3's integrated $\mathrm{CN}-g^{\prime}$ color seen in Figure 8. It is important to note, however, that M3's initial central concentration of SG stars is unknown.

M5's present-day median relaxation time of $2.6 \mathrm{Gyr}$ (Harris 2010) is less than a quarter of its physical age (11.5 Gyr; VandenBerg et al. 2013). It therefore represents an intermediate case and evidence for its population gradient is ambiguous. Histograms of the individual stars' $\Delta\left(\mathrm{CN}-g^{\prime}\right)$ index (Figure 5), divided equally into three radial bins, show a wider distribution of $\Delta\left(\mathrm{CN}-g^{\prime}\right)$ color in the inner bin but a similar average color compared to the outer two bins. The ECDFs (Figure 7) for $\mathrm{CN}$-weak and for $\mathrm{CN}$-strong stars are similar, indicating little difference in the central concentration of its first and SG stellar populations. The population ratio plot given in Figure 6 also shows no change in the relative numbers of first and SG stars with radius. The overall $\mathrm{CN}-g^{\prime}$ color gradient for M5 (shown in Figure 8) is flat to $\sim 1.5$ half-light radii and then may decline slightly at larger radii.

Our ECDFs for M5 are consistent with an analysis of $\mathrm{Na}$ abundances by Cordero (2014), including both her own measurements and those of Ramírez \& Cohen (2002). As Cordero (2014) notes, the similarity in the cumulative distribution functions of first and SG stars is discrepant with the analysis of $\left(u^{\prime}-g^{\prime}\right)$ colors from SDSS photometry by Lardo et al. (2011), who find the SG to be more centrally concentrated than the FG population. Cordero suggests that Lardo et al.'s sample better represents the stellar populations within one half-mass radius, while the spectroscopic data better sample the cluster at larger radii.

At the age and current relaxation time of M5, any initial difference between central concentrations of first and SG stars will be significantly reduced. If the difference was initially high, it would have largely been erased in the present-day cluster. If the difference was initially small, it could persist longer, but would be difficult to detect observationally. After more than four dynamical timescales, the fraction of SG stars would be flat to beyond one half-light radius and then decline slowly. This behavior is consistent with our observation of the change in $\mathrm{CN}-g^{\prime}$ integrated color out to 2.5 half-light radii (Figure 8).

The present-day median relaxation time for M13 is about 2 Gyr (Harris 2010), which is one-sixth of its 12 Gyr lifetime (VandenBerg et al. 2013). As we found for M5, histograms of the $\Delta\left(\mathrm{CN}-g^{\prime}\right)$ color in radial bins (Figure 5) show a broader distribution of stars in the central bin, but the mean color in the three bins is similar within our observational uncertainty. The population ratios in Figure 6 show an excess of $\mathrm{CN}$-rich stars within $r_{e}<1$, and the ECDF curves for $\mathrm{CN}$-rich and $\mathrm{CN}$-poor stars in Figure 7 suggest that $\mathrm{CN}$-rich stars are somewhat more centrally concentrated. In integrated light (Figure 8), the radial dependence of the $\mathrm{CN}-g^{\prime}$ color is flat within 1.5 half-light radii, and, as in $\mathrm{M} 5$, the $\mathrm{CN}-g^{\prime}$ color declines slightly outside that radius.

Johnson \& Pilachowski (2012) examined the radial distributions of primordial, intermediate, and extreme stars in M13, finding relative populations of $15 \%, 63 \%$, and $22 \%$, respectively, in their sample. They found that the extreme population is highly centrally concentrated, while the radial distributions of the primordial and intermediate populations are similar. In order to compare more directly to our photometry, we have resorted the Johnson \& Pilachowski (2012) sample into two similarly sized subpopulations based simply on the stars' $\mathrm{Na}$ abundances. We find little difference in the radial concentrations of the Na-rich and Na-poor stars when the sample is divided into equal halves. Moreover, the cumulative distribution functions from the $\mathrm{Na}$ abundance data are similar to what we find for our $\Delta\left(\mathrm{CN}-g^{\prime}\right)$ photometry when the stellar samples are divided into nearly equal halves.

While the clear central concentration of M13's extreme population persists (Johnson \& Pilachowski 2012), its intermediate and primordial populations are well mixed, exhibiting virtually no difference in central concentration. While the extreme population in M13 is highly centrally concentrated (Johnson \& Pilachowski 2012), primordial and intermediate stars still dominate within $r_{e}<1$ due to the relatively small fraction of extreme stars in the cluster $(\sim 15 \%)$. As is the case for M5, either the initial difference in the central concentrations of M13's intermediate and primordial populations has been reduced by dynamical evolution within 1.5 half-light radii, or the central concentrations of these populations were similar to begin with. Beyond 1.5 half-light radii, the $\mathrm{CN}-g^{\prime}$ color declines slightly, consistent with M13's dynamical age. The lack of evidence for radial gradients in M5 and M13 is also consistent with the results from the study of the distribution of extreme blue and red horizontal branch stars in these clusters (Vanderbeke et al. 2015).

M71, at an age of 11 Gyr (VandenBerg et al. 2013) and with a present-day dynamical relaxation time of $0.84 \mathrm{Gyr}$ (Harris 2010), is the dynamically oldest cluster in our sample. Its central radial bin does not contain an excess of $\mathrm{CN}$-strong stars (Figure 5), its ECDF shows no evidence for CN-strong stars being centrally concentrated (Figure 7), the population ratio gives no hint of segregation in the distribution of SG stars (Figure 6), and there is no radial gradient in the cluster's integrated $\mathrm{CN}-g^{\prime}$ color (Figure 8). These results are consistent 
with the observations of Cordero et al. (2015), who found no statistical difference in the central concentrations of first and SG stars in M71, as identified from their analysis of $\mathrm{Na}$ abundances in 75 cluster members.

At the advanced dynamical age of M71, the simulations of Vesperini et al. (2013) suggest that the cluster should be well mixed, with no central concentration of its SG stars, just as observed. Vesperini et al. (2013) also note that as a cluster approaches complete mixing, statistical fluctuations can add noise into the ECDF curves leading to small but detectable differences between populations, particularly in regions beyond 1-2 half-mass radii. This effect could explain the separation of the $\mathrm{CN}$-weak and CN-strong curves for M71 in Figure 7.

Overall, the $\Delta\left(\mathrm{CN}-g^{\prime}\right)$ index provides an effective tool for exploring the radial distributions of stellar populations in GCs. In each of the four clusters, M3, M5, M13, and M71, the index correlates with spectroscopically measured $[\mathrm{Na} / \mathrm{Fe}]$ ratios and qualitatively reproduces published ECDFs for first and SG stars. Integrated light observations using a narrow-band filter at the $\mathrm{CN}-\lambda 3883$ band allow the identification of stellar population gradients in clusters, with potential application to clusters where photometric measurements of individual stars may not be practical. Combined with broadband photometric data from all-sky surveys such as SDSS (An et al. 2008) and Pan-STARRS (Chambers et al. 2016), and eventually from the Large Synoptic Survey Telescope, observations with a single narrow-band filter can identify and characterize stellar population gradients in large numbers of clusters.

\section{Summary}

A fuller understanding of the formation of GCs requires studying these systems in galaxies with different formation histories. Once outside the Galaxy, spectroscopic measurements of individual stars are not possible-one will have to probe for stellar population differences with integrated light. Our analysis of the GCs M3, M5, M13, and M71 demonstrates that $\mathrm{CN}-g^{\prime}$ photometry is a viable way of making these measurements. There is a rich history of using the blue $\mathrm{CN}$ absorption feature to separate stellar populations, both spectroscopically and photometrically. Furthermore, changes in the $\mathrm{CN}$-band strengths can be observed at low resolution and even photometrically, while other indicators like sodium or oxygen abundances require high-resolution spectra.

When we compare $\mathrm{CN}-g^{\prime}$ colors with spectroscopically determined $[\mathrm{Na} / \mathrm{Fe}]$ measurements for these four nearby GCs, we find clear evidence for a strong correlation between the two quantities. This relationship establishes that we can use the two-filter system to distinguish stellar populations, and indeed, when we examine the systems' RGBs, we find that all four have a spread in color that is many times broader than that associated with the photometric errors. Moreover, in the dynamically young, intermediate-metallicity cluster M3, we find evidence for a radial dependence in both the mean and the dispersion of the $\mathrm{CN}-g^{\prime}$ color distribution, in agreement with previous measurements (e.g., Carretta et al. 2010). The situation is less clear for M5, for which the evidence for a gradient in $\mathrm{CN}$ strength as a function of radius is ambiguous. For M13, the primordial and intermediate populations appear to be well mixed, although the extreme population is centrally concentrated. No gradient is seen in M71, though given its old dynamical age, this result is unsurprising.
Finally, we examined the relationship between $\mathrm{CN}-g^{\prime}$ measurements for individual RGB stars and similar measurements for integrated GC light. We showed that for M3, integrated light measurements can detect the known $\mathrm{CN}-g^{\prime}$ color gradient, and that the effect of stellar stochasticity on the measurements is small in all systems, with an integrated absolute $g^{\prime}$ magnitude brighter than $g^{\prime} \sim-5$. Future efforts will seek to apply this integrated light analysis to GCs in nearby galaxies and allow us to better understand how subpopulations impact the formation and evolution of these systems. For example, the use of an integrated light approach should prove useful for probing multiple populations in M31 GCs, especially clusters from the PAndAS catalog (Huxor et al. 2014) with half-light radii $\left(r_{e}\right)$ exceeding $10^{\prime \prime}$. In these systems, integrated $\mathrm{CN}-g^{\prime}$ color gradients should allow us to detect population variations within clusters over a wide range of mass, dynamical age, and galactocentric radius. Population ratios can then be obtained from integrated color gradients in combination with detailed population synthesis.

We are grateful to the anonymous referee for her/his critical and constructive comments and for helping to improve the overall quality of this study. We thank Enrico Vesperini, Alex Hagen, Joanna Bridge, and Owen Boberg for their help, insight, and thoughtful comments as we prepared this manuscript. The $0.9 \mathrm{~m}$ telescope is operated by WIYN Inc. on behalf of a Consortium of partner Universities and Organizations (see http://www.noao.edu/0.9m for a list of the current partners). WIYN is a joint partnership of the University of Wisconsin at Madison, Indiana University, Yale University, and the National Optical Astronomical Observatory. W.P.B. and C.A.P. gratefully acknowledge support from the Daniel Kirkwood endowment at Indiana University. The Institute for Gravitation and the Cosmos is supported by the Eberly College of Science and the Office of the Senior Vice President for Research at the Pennsylvania State University. This research has made use of the VizieR catalog access tool, CDS, Strasbourg, France. The original description of the VizieR service was published in A\&AS 143, 23. This research also made use of the USNOFS Image and Catalog Archive operated by the United States Naval Observatory, Flagstaff Station (http://www.nofs. navy.mil/data/fchpix/). This research has made use of NASA's Astrophysics Data System Bibliographic Services.

\section{ORCID iDs}

William P. Bowman (iD https://orcid.org/0000-00034381-5245

Catherine A. Pilachowski (i) https://orcid.org/0000-00023007-206X

Robin Ciardullo (iD https://orcid.org/0000-0002-1328-0211

\section{References}

\footnotetext{
Abazajian, K. N., Adelman-McCarthy, J. K., Agüeros, M. A., et al. 2009, ApJS, 182, 543

An, D., Johnson, J. A., Clem, J. L., et al. 2008, ApJS, 179, 326

Bedin, L. R., Piotto, G., Anderson, J., et al. 2004, ApJL, 605, L125

Calamida, A., Bono, G., Stetson, P. B., et al. 2007, ApJ, 670, 400

Carretta, E., Bragaglia, A., Gratton, R., \& Lucatello, S. 2009a, A\&A, 505, 139

Carretta, E., Bragaglia, A., Gratton, R. G., et al. 2010, A\&A, 516, A55

Carretta, E., Bragaglia, A., Gratton, R. G., et al. 2009b, A\&A, 505, 117

Chambers, K. C., Magnier, E. A., Metcalfe, N., et al. 2016, arXiv:1612.05560

Cordero, M. J. 2014, PhD thesis, Indiana Univ.
} 
Cordero, M. J., Pilachowski, C. A., Johnson, C. I., \& Vesperini, E. 2015, ApJ, 800, 3

Cummings, J. D., Geisler, D., Villanova, S., \& Carraro, G. 2014, AJ, 148, 27

Davis, L. E. 1994, in ASP Conf. Ser. 61, Astronomical Data Analysis Software and Systems III, ed. D. R. Crabtree, R. J. Hanisch, \& J. Barnes (San Francisco, CA: ASP), 75

Feigelson, E. D., \& Babu, G. J. 1992, ApJ, 397, 55

Gratton, R. G., Carretta, E., \& Bragaglia, A. 2012, A\&ARv, 20, 50

Harris, W. E. 2010, arXiv:1012.3224

Hsyu, T., Johnson, C. I., Lee, Y.-W., \& Rich, R. M. 2014, PASP, 126, 597

Huxor, A. P., Mackey, A. D., Ferguson, A. M. N., et al. 2014, MNRAS, 442,2165

Johnson, C. I., Kraft, R. P., Pilachowski, C. A., et al. 2005, PASP, 117, 1308

Johnson, C. I., \& Pilachowski, C. A. 2012, ApJL, 754, L38

Kamann, S., Wisotzki, L., Roth, M. M., et al. 2014, A\&A, 566, A58

Kayser, A., Hilker, M., Grebel, E. K., \& Willemsen, P. G. 2008, A\&A, 486, 437

Kimmig, B., Seth, A., Ivans, I. I., et al. 2015, AJ, 149, 53

Langer, G. E. 1985, PASP, 97, 382

Lardo, C., Bellazzini, M., Pancino, E., et al. 2011, A\&A, 525, A114

Lee, J.-W. 2015, ApJS, 219, 7

Lee, Y.-W., Han, S.-I., Joo, S.-J., et al. 2013, ApJL, 778, L13

Lim, D., Han, S.-I., Lee, Y.-W., et al. 2015, ApJS, 216, 19

Martell, S. L., \& Smith, G. H. 2009, PASP, 121, 577

Massari, D., Lapenna, E., Bragaglia, A., et al. 2016, MNRAS, 458, 4162

Milone, A. P., Piotto, G., Bedin, L. R., et al. 2012, ApJ, 744, 58
Milone, A. P., Marino, A. F., Piotto, G., et al. 2015, ApJ, 808, 51

Milone, A. P., Piotto, G., Renzini, A., et al. 2016, MNRAS, 455, 3009

Milone, A. P., Piotto, G., Renzini, A., et al. 2017, MNRAS, 464, 3636

Monet, D. G., Levine, S. E., Canzian, B., et al. 2003, AJ, 125, 984

Piotto, G., Milone, A. P., Bedin, L. R., et al. 2015, AJ, 149, 91

Ramírez, S. V., \& Cohen, J. G. 2002, AJ, 123, 3277

Roh, D.-G., Lee, Y.-W., Joo, S.-J., et al. 2011, ApJL, 733, L45

Samus, N. N., Kazarovets, E. V., Pastukhova, E. N., Tsvetkova, T. M., \& Durlevich, O. V. 2009, PASP, 121, 1378

Sbordone, L., Salaris, M., Weiss, A., \& Cassisi, S. 2011, A\&A, 534, A9

Smith, G. H. 2015a, PASP, 127, 825

Smith, G. H. 2015b, PASP, 127, 1204

Smith, G. H., \& Briley, M. M. 2006, PASP, 118, 740

Smith, G. H., \& Langland-Shula, L. E. 2009, PASP, 121, 1054

Smith, G. H., Modi, P. N., \& Hamren, K. 2013, PASP, 125, 1287

Stetson, P. B. 1987, PASP, 99, 191

Strömgren, B. 1966, ARA\&A, 4, 433

Suntzeff, N. B. 1981, ApJS, 47, 1

VandenBerg, D. A., Brogaard, K., Leaman, R., \& Casagrande, L. 2013, ApJ, 775,134

Vanderbeke, J., De Propris, R., De Rijcke, S., et al. 2015, MNRAS, 451 275

Vesperini, E., McMillan, S. L. W., D’Antona, F., \& D’Ercole, A. 2013, MNRAS, 429, 1913

Villanova, S., Piotto, G., King, I. R., et al. 2007, ApJ, 663, 296

Yong, D., Grundahl, F., Johnson, J. A., \& Asplund, M. 2008, ApJ, 684, 1159 